\title{
Plädoyer für ein funktionales Basismodell gemeindepsychiatrischer Versorgung
}

\section{A Plea for a Functional Basic Model of Psychiatric Care}

Autoren

Institute
Ingmar Steinhart ${ }^{1}$, Günther Wienberg ${ }^{2}$

Institut für Sozialpsychiatrie Mecklenburg-Vorpommern, Ernst-Moritz Arndt-Universität Greifswald

${ }^{2}$ Hochschule Fulda, Fachbereich Sozialwesen

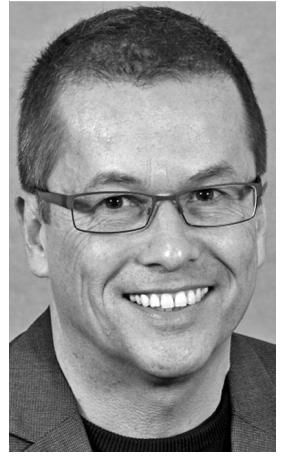

Ingmar Steinhart

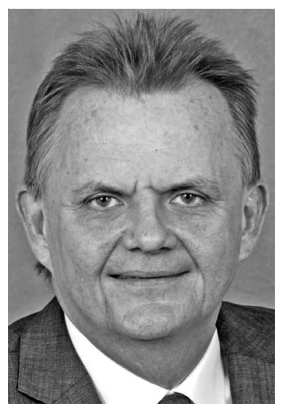

Günther Wienberg

\section{Bibliografie}

Dol http://dx.doi.org/ 10.1055/s-0034-1370023 Psychiat Prax 2014; 41:

179-181

(c) Georg Thieme Verlag KG Stuttgart · New York

ISSN 0303-4259

\section{Korrespondenzadresse}

Prof. Dr. Ingmar Steinhart Institut für Sozialpsychiatrie Mecklenburg-Vorpommern, An-Institut der Ernst-Moritz Arndt-Universität Greifswald Ellernholzstraße 1-2 17487 Greifswald institut@sozialpsychiatrie-mv.de
Die Psychiatriereform kann als eines der erfolgreichsten und nachhaltigsten sozialen Reformprojekte der letzten Jahrzehnte beschrieben werden [1]. So ist es zu einem erheblichen Bettenabbau in der Regelversorgung gekommen: von 117600 1972 auf 52000 in 2005. Dies ist vor allem der Enthospitalisierung chronisch psychisch Kranker in Verbindung mit den neuen Möglichkeiten der Pharmakotherapie geschuldet. Seit 2006 ist allerdings eine starke Tendenz zur erneuten Ausweitung stationärer und teilstationärer Kapazitäten zu verzeichnen, die Bettenzahl stieg bis 2012 bundesweit um ca. 1100, die Zahl der Tagesklinikplätze zwischen 2000 und 2010 um 43\% [2]. Institutsambulanzen und niedergelassene Psychotherapeuten verzeichnen stark steigende Fallzahlen. Diese Leistungsausweitung ist nicht bedingt durch eine gestiegene Prävalenz psychischer Störungen in der Population [3, 4]. Gestiegen ist demnach die Inanspruchnahme der psychiatrisch-psychotherapeutischen Versorgung (PPV). Die Psychiatrie debattiert derweil über Chancen und Risiken des PEPP-Systems. Manche möchten das bisherige Vergütungssystem in die Zukunft fortschreiben, andere versuchen, die Flucht in eine Modellvariante nach $\S 64$ b SGB V anzutreten. Dabei scheint es eher um den ökonomischen Bestandsschutz zu gehen und weniger darum, die nach wie vor gravierenden Strukturprobleme der PPV zu lösen:

- Leistungsbedarf und Ressourcenzuweisung verhalten sich teilweise umgekehrt proportional zueinander. So versorgen z.B. die niedergelassenen Fachärzte für Psychiatrie etwa 72\% der Patienten mit 26\% des Budgets, während Psychotherapeuten 28\% der Patienten mit 74\% des Budgets behandeln [5].

- Behandlungsdichte und Behandlungssetting sind eng verknüpft. Das heißt, dass der Bedarf nach zeitintensiver und komplexer psychiatrischer Behandlung nur unter stationären Bedingungen gedeckt werden kann und dass
Veränderungen der benötigten Behandlungsdichte regelhaft mit einem Wechsel des Behandlungssettings einhergehen.

- Belegte Betten bzw. Plätze sind immer noch leichter finanzierbar als aufwendige ambulante Therapien.

- Strukturelle Antworten auf die Anforderungen aus den neuen S3-Leitlinien und der UN-Behindertenrechts-Konvention (BRK) bleiben aus.

Eklatante Versorgungs- und Finanzierungslücken bestehen fort. Eine Kalkulation der GKV-finanzierten Tageskosten gängiger Behandlungsangebote auf der Basis von 7 Tagen/Woche und 30 Tagen/Monat ergibt: Niedergelassene Psychiater (ca. $70 € /$ Quartal) weniger als $1 € /$ Tag; PIA (ca. $360 € /$ Quartal) ca. $4 € /$ Tag; niedergelassene Psychotherapeuten (ca. $80 € /$ Woche) ca. $11 € /$ Tag; Tagesklinik (ca. $160 € /$ Tag für 5 Tage) ca. $107 € /$ Tag; stationäre Behandlung $\geq 240 € /$ Tag. Die tagesklinische Regelbehandlung ist also 10-mal teurer wie die aufwendigste ambulante! Auch zwischen teil- und vollstationärer Behandlung gibt es eine Lücke von ca. $100 €$. Auf diese Weise sind Patienten mit intensiven und komplexen Behandlungsbedarfen regelhaft auf die stationäre und damit teuerste und eingreifendste Behandlung verwiesen.

Angesichts dieser Probleme greift nüchterner Pragmatismus im Umgang mit PEPP zu kurz. Gefragt ist eine attraktive Zukunftsvision für die PPV. Diese kann nur im ambulanten Bereich liegen, also genau dort, wo PEPP - abweichend vom ursprünglichen Auftrag des Gesetzgebers - gar nicht erst hin reicht!

Als Zielmarke für die Weiterentwicklung der PPV schlagen wir ein funktionales Basismodell der Versorgung vor. Funktional heißt, dass diese Funktionen in jeder Versorgungsregion obligatorisch sicherzustellen sind, unabhängig von der jeweiligen organisatorischen Umsetzung. Das Modell orientiert sich an grundlegenden Bestimmungen 


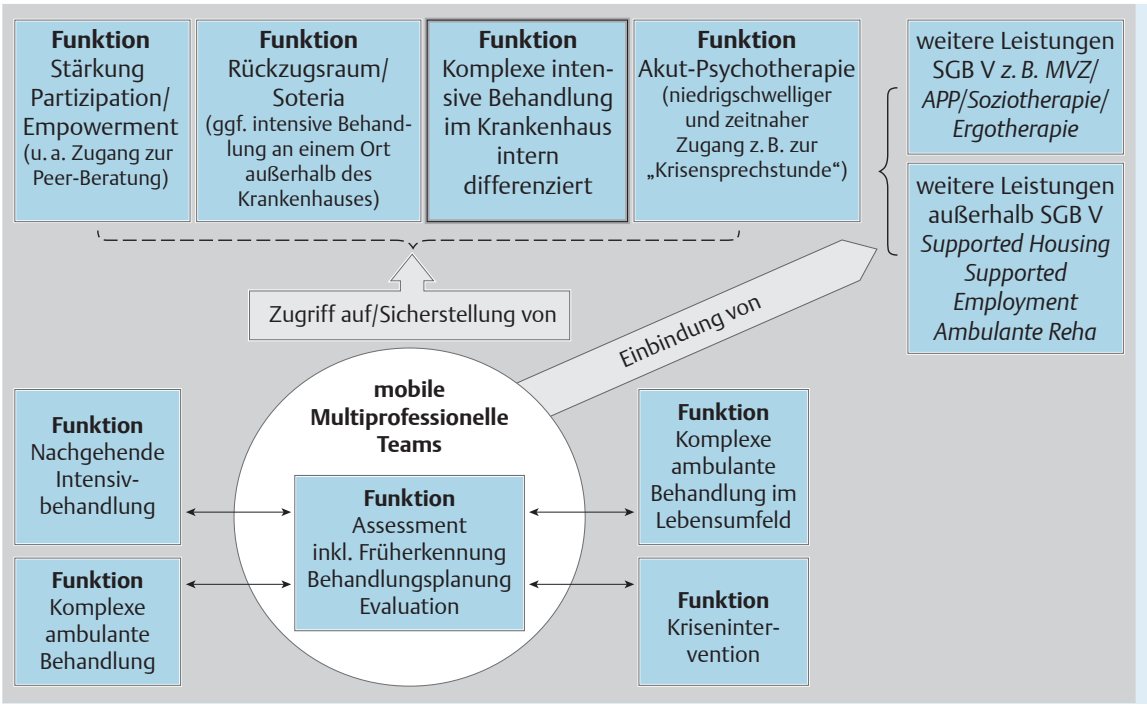

Abb. 1 Das im Rahmen der GKV zu finanzierende Basismodell Gemeindepsychiatrie stellt die Sicherstellung der Kernfunktionen ambulanter mobiler multiprofessioneller Teams in das Zentrum. Neue Funktionen sind der niedrigschwellige Zugang zu Akutpsychotherapie, Peer-Beratung und zu SoteriaAngeboten/Rückzugsräumen. der BRK sowie an der verfügbaren wissenschaftlichen Evidenz. Die BRK fordert in Artikel 19 die Barrierefreiheit von Hilfen, insbesondere den „Zugang zu einer Reihe von gemeindenahen Unterstützungsdiensten zu Hause“. Außerdem ist die aktuelle S3Leitlinie „Psychosoziale Therapien bei schweren psychischen Erkrankungen“ [6] wegweisend. Sie unterscheidet zwischen einzelfallbezogenen und systembezogenen Interventionen. Letztere sind strukturelle oder prozessbezogene Versorgungsmerkmale unabhängig von spezifischen Störungsbildern. Sie beruhen überwiegend auf Forschungsergebnissen aus dem angloamerikanischen Raum, denn die deutsche Versorgungsforschung weist erhebliche Defizite auf [7]. Die Evidenz bezieht sich vor allem auf Community Mental Health Teams [8], das Assertive Community Treatment [11] und das Home Treatment [10]. Zu diesen nicht trennscharfen Modellen liegen inzwischen international weit mehr als 100 RCTStudien vor. Gemeinsame Merkmale sind Multiprofessionalität, Teambasierung, regionaler Versorgungsauftrag und die Zielgruppe schwer psychisch kranke Personen, für die die Behandlung durch Therapeuten in freier Praxis nicht ausreicht. Unterschiede beziehen sich auf Mobilität, Fallzahl je Fachkraft, zeitliche Erreichbarkeit und Akuität der Erkrankung. Alle 3 Modelle weisen insgesamt signifikante Vorteile bei der Reduzierung von Zahl bzw. Dauer stationärer Behandlungen, bei der Reduzierung von Behandlungsabbrüchen, bei der Zufriedenheit von Patienten und Angehörigen sowie bei der Kosteneffektivität auf [6].

Das Basismodell bezieht sich auf die Versorgung von Menschen mit schweren und komplexen psychischen Störungen, die nicht ausreichend durch niedergelassene Fachärzte und Psychotherapeuten behandelt werden können ( $\bullet$ Abb.1). Grundbaustein sind multiprofessionelle und mobile Teams (MMT), die folgende Funktionen abdecken:

- komplexe ambulante Behandlung (analog CMHT)

- komplexe Behandlung im Lebensumfeld (analog HT)

- nachgehende Intensivbehandlung (analog ACT)

- Krisenintervention und Erreichbarkeit an 24 Stunden/7 Tagen

- Gatekeeping für andere Versorgungsbausteine durch qualifiziertes Assessment, Behandlungsplanung und -evaluation

- Behandlung in einem krankenhausalternativen Setting (Rückzugsraum, Soteria-Milieu)

- niedrigschwelliger Zugang zu ambulanten psychotherapeutischen Leistungen („Akut-Psychotherapie“)
- Sicherstellung des Zugangs zu Peer to Peer-Beratung durch geschulte Psychiatrieerfahrene und Angehörige (psychiatrische Dienste unter Beteiligung von Psychiatrieerfahrenen weisen zumindest keine schlechteren Ergebnisse auf als rein professionelle Dienste [11, 12]).

- Integration ergänzender Funktionen: ambulante psychiatrische Pflege, Soziotherapie, ambulante Ergotherapie

- Erschließung von Leistungen außerhalb des SGB V: ambulante Reha, „supported housing“ und „supported employment“.

Das Basismodell deckt sämtliche Funktionen der PPV von Menschen mit schweren und komplexen Störungen ab. Dabei wird die gesamte Behandlung von der ambulanten Seite aus gedacht, geplant und gesteuert. Auch der Zugang zu (teil)stationärer Behandlung erfolgt über die MMT und die Patienten werden bei (teil)stationärer Episoden idealerweise durch das MMT (mit)behandelt.

Das Finanzierungssystem unterstützt ein solches ambulant gestütztes Behandlungsmodell bisher nicht ausreichend. In den letzten Jahren haben sich jedoch bundesweit diverse Modelle der krankenhausersetzenden Behandlung entwickelt, die Elemente des Basismodells umsetzen [13].

Es ist problematisch, dass die in Deutschland aktuell verhandelten und diskutierten Modelle nach $§ 64$ b SGB V nicht primär an einem fachlich begründeten Strukturmodell der PPV ausgerichtet sind, wie wir es auf der Basis der BRK und der verfügbaren Evidenz hier vorschlagen. Die Schnittstellen in der Kooperation und bei der Steuerung der Finanzressourcen zu anderen Leistungsanbietern, insbesondere zu Fachärzten und zum Leistungsgeschehen im SGB XII stehen bisher ebenso wenig im Fokus wie die Stärkung der Nutzerbeteiligung. Der Umstand, dass die Einführung innovativer ambulanter Behandlungsmodelle die Inanspruchnahme stationärer Versorgung wahrscheinlich nur dann reduziert, wenn Betten knapp sind bzw. wenn die Innovation mit einer Bettenreduktion strukturell verknüpft wird [14], hält bisher offenbar viele Leistungserbringer und -träger von einer forcierten Umsetzung ambulant gestützter Modelle ab. Auch die Transparenz des Leistungsgeschehens ist nicht immer gegeben (z.B. bei Modellen mit regionalen Krankenhausbudgets).

Trotz dieser Herausforderungen können PEPP oder etwaige Modifikationen nicht das Ende aller Überlegungen sein. Modelle mit eindeutig ambulantem Schwerpunkt sind stärker als bisher zu fördern, umzusetzen und zu evaluieren. Für solche Modelle 
schafft unser Vorschlag eine fachliche Plattform. Künftige Forschungsanstrengungen müssen darauf gerichtet sein, unterschiedliche Umsetzungsvarianten vergleichend $\mathrm{zu}$ evaluieren. Anzustreben sind Multicenterstudien, die von vornherein auch Kosten-Effektivitätsaspekte einschließen.

\section{Literatur}

1 Wienberg G. 40 Jahre Psychiatriereform in Deutschland - Auf dem Weg in die Drei Klassen-Psychiatrie? Sozialpsychiatr Inform 2014; 44: 4-9

2 Gesundheitsministerkonferenz - Arbeitsgruppe Psychiatrie. Weiterentwicklung der psychiatrischen Versorgungstrukturen in Deutschland - Bestandsaufnahe und Perspektiven. Im Internet: www.ham burg.de/krankenhaus/3506156/gmk-beschluss.html; Stand: 19.1.2014

3 Richter D, Berger K. Nehmen psychische Störungen zu? Update einer systematischen Übersicht über wiederholte Querschnittsstudien Psychiat Prax 2013; 40: 176-182

4 Jacobi F, Höfler M, Strehle J et al. Psychische Störungen in der Allgemeinbevölkerung. Studie zur Gesundheit Erwachsener in Deutschland und ihr Zusatzmodul Psychische Gesundheit (DEGS1-MH). Nervenarzt 2014; 85: 77-87

5 Melchinger H. Schwierigkeiten, Verteilungsprobleme, Leistungsstrukturen und Bedarf in der Versorgung. Vortrag Tagung „Psychosen - Persönliches Leiden und gesellschaftliche Realität“". Ev. Akademie Loccum; 2013
6 DGPPN, Hrsg. S3-Leitlinie Psychosoziale Therapien bei Menschen mit schweren psychischen Erkrankungen. Heidelberg: Springer; 2012

7 Kilian R. Gesundheitsökonomische Evaluation gemeindepsychiatrischer Interventionen. Nervenarzt 2012; 83: 832 -839

8 Department of Health. Mental Health Policy Implementation Guide Community Mental Health Teams 2002. Im Internet: webarchive. nationalarchives.gov.uk/20130107105354/htpp:/www.dh.gov.uk/ prod_consum_dh/groups/dh_digitalassets/@ps/documents/digitalas sets/dh_085652.pdf (Stand: 01.04.2014)

9 Assertive Community Treatment. Im Internet: www.actassociation. org/ActModel (Stand: 19.1.2014)

10 Gühne $U$, Weinmann S, Arnold $K$ et al. Akutbehandlung im häuslichen Umfeld: Systematische Übersicht und Implementierungsstand in Deutschland. Psychiat Prax 2011; 38: 114-122

11 Doughty C, Tse S. Can consumer-led mental health services be equally effective? An integrative review of CLMH services in high-income countries. Community Ment Health J 2011; 47: 252 - 266

12 Pitt V, Lowe D, Hill S et al. Consumer-providers of care for adult clients of statutory mental health services. Cochrane Database Syst Rev 2013; 3: CD004807

13 Steinhart I, Wienberg G, Koch C. Krankenhausersetzende psychiatrische Behandlung in Deutschland - Praxismodelle, Standards und Finanzierung. Psychiat Prax [eingereicht]

14 Weinmann S, Gühne $U$, Kösters $M$ et al. Teambasierte Gemeindepsychiatrie. Nervenarzt 2012; 83: 825-831 